Original Article

\title{
Executive Functioning in Healthy Aged Population in comparison to Young Adults Using Cognitive Estimation Test
}

\section{Shrunga M S ${ }^{1}$, Livingston Sengolraj ${ }^{2}$, Radish Kumar B. ${ }^{3}$}

${ }^{1}$ Lecturer, Nitte Institute of Speech and Hearing, Mangalore, ${ }^{2}$ Audiologist, Branch Manager and Microsuction earwax Specialist, Amplifon, United Kingdom, ${ }^{3} \mathrm{Head}$, Department of Audiology and Speech Language Pathology, Kasturba M edical College, Mangalore.

Corresponding Author: Shrunga M S, Lecturer, Nitte Institute of Speech and Hearing, M angalore, Karnataka E-mail : msshrunga@gmail.com.

Received : :22.02.2018

Review Completed : 31.05.2018

Accepted : : 12.06.2018

Keywords : Cognition, Executive Functions, cognitive estimation

Access this article online Quick Response Code

\begin{abstract}
:
Background and objective: Cognitive estimation is an important task in which people engage themselves in estimating the environment in different aspects of their life, such as; distance, quantity, size, weight etc The study was taken up to evaluate the cognitive estimation skills in Healthy Aged Population in comparison to Young Adults.

Methodology: A total of 40 healthy young and old adults participated in the study. The participants were divided into two groups. Cognitive Estimation Test (CET) was administered for both the groups. The CET, was modified to suit Indian population consisted of 18 questions under the domains of distance, quantity, size, weight, temperature and time. Responses were scored for Under-estimation (UE), Over-estimation (OE), Approximate answer (AA), and No answer (NA). Number of UE, OE, AA, and NA were calculated. Independent t-test was employed to find out the significant differences between the means of both the groups.
\end{abstract}

Results: Results revealed that young adults were better in estimating the environment when compared to healthy aged population, which was significant .Distance and size dimensions were remarkably affected in comparison to all other dimensions. Instances of No answers (NA) were higher in healthy aged population in comparison to young adults.

Conclusion: The results indicate that, the decline in cognitive estimation abilities is a part of healthy aging rather than a pathological condition. Thus CET can be used as a tool in the assessment and management of cognitive disorders.

\section{Introduction}

Cognitive estimation is an important function in daily life in which people engage themselves in estimating the environment in different aspects of their life. Cognitive estimation involves the attempt to provide reasonable answers to questions for which relevant knowledge, but not the specific answer, is available to the subject ${ }^{[1]}$.It measures the person's ability to estimate different aspects such as distance, time, weight, size etc. A different kind of reasoning is employed in these tasks where inferences about the world are drawn from known information. For example, people were asked to answer certain questions with unknown but approximately estimable answers such as 'How long will a person take to climb 20 steps?' or 'How heavy will be a 100 pages book?' The effective estimation of such tasks requires good relation between mapping and metric properties. In order to provide a reasonable estimate, the subject must be able to identify the relevant knowledge set, retrieve specific facts or estimates from within that set, devise and carry out appropriate manipulations on those facts or estimates, project the result against his/her knowledge of the world to judge its reasonableness or probability, and repeat any necessary part of the sequence to produce a better response. Occasionally individuals may not have the exact estimation ability of a particular object (Eg: length of the wall), but still able to approximate the correct answer with reference to the knowledge of other known object (Eg: their own height). Linguistic tasks are commonly used to assess the cognitive status of an individual. But, there are 
consensuses that whether these tasks measure the composite abilities that draw on multiple cognitive domains, including working memory and, executive functions ${ }^{[2]}$.

Adequate performance in cognitive estimation utilizes frontal executive function, semantic memory, as well as other cognitive domains. Executive functions allow individuals to evaluate their situation in order to achieve their goal, and then carry out a plan to achieve that goal and include processes such as problem solving, decision making, planning, initiation, inhibition and monitoring. These functions are thought to play an important role in CET performance. It is also found that functional brain imaging studies of executive control processes report robust differences in brain activity between older and younger subjects, particularly under conditions of high executive control demand ${ }^{[3]}$.

Several estimation tests have been previously developed, but these lacked standardized scoring methods ${ }^{[4]}$.The Cognitive Estimation Test (CET) represents a novel diagnostic tool for quantifying the ability to provide accurate cognitive estimates. Cognitive estimates are supposed to result from frontal cortex activity. Cognitive Estimation Test was originally designed to examine the estimating abilities ${ }^{[5]}$. Authors of CET were the first investigators to draw attention to a particular cognitive deficit, namely the 'gross inability to produce adequate cognitive estimates'. Cognitive Estimation Test was developed to quantify the ability to provide accurate cognitive estimates in patients with frontal lobe damage ${ }^{[5]}$. $M$ any types of brain dysfunction will produce deficits in the final estimation, and frontal lobe dysfunction is especially likely to produce such deficits ${ }^{[5,6]}$.

Aging is a continuous process which is nothing but the accumulation of changes in a person over time and it is referred to a multidimensional process of physical, psychological, and social change. Aging may affect topdown processing, use of prior knowledge, and other elementary processes which take part in a variety of cognitive abilities. Such changes may cause older people to seem as if they have declined in all cognitive processes. The process of aging is not a single dimension progression, but a complex of strands, or paths of concern, continuing for different lengths of time throughout a life's biography and moulding its individuality. Some dimensions of aging grow and expand over time, while others decline ${ }^{[7]}$. Aging is often associated with changes in cognitive capacity. It is accompanied by significant structural alterations in the basic elements of CNS - the neurons. Brain volume and probably, in the number of active neurons, the age dependent drop in metabolic activity may be related to loss of working tissue. Since brain undergoes structural and functional changes during healthy aging, it is expected that they perform poorly on cognitive estimation tasks in comparison to young adults. The present study was aimed at investigating differences if any in the cognitive estimation task between the healthy aged population and the young adults.

\section{Need for the study}

With the review of literature, it is clear that aging is often associated with changes in the cognitive capacity. In the present study, it was hypothesized that healthy aged population would perform poorly on the test of cognitive estimation $\&$ hence present study was taken up with the aim of investigating the cognitive estimation skills in a healthy aged population in comparison to young adults.

\section{Aim of the study}

The aim of the study was to evaluate the cognitive estimation skills in Healthy Aged Population in comparison to Young Adults.

\section{Materials and Method}

Study Design: Prospective observational study Participants

The present study included a total of 40 participants. The participants were divided into two groups. Group one consisted of twenty normal young adults in the age range of $18-25$ years. Group two consisted of twenty healthy aged populations in the age range of 60-80 years. Group one participants are students from a medical college and group two participants are normal elderly individuals 
participating in activities at a senior center. All the participants were educated with a minimum qualification of 12th standard and belong to middle-higher socio economic status. All participants had no history of existing speech, language, neurological or psychiatric illness. An informed consent was taken from all participants before the commencement of the study.

\section{Materials}

Cognitive Estimation Test (CET) ${ }^{[5]}$ was administered for both the groups of participants. The CET, which was modified to suit Indian population, consisted of 18 questions under the domains of distance(3), quantity(3), size(3), weight(3), temperature(2) and time(4). Proper units for these dimensions were given for each question. Modified CET test is available in AppendixA.

\section{Procedure}

The participants were seated in a comfortable chair. Questionnaire was given to the participants in a printed format, with options given for each question. Responses were scored for Under-estimation (UE), Over-estimation $(\mathrm{OE})$, Approximate answer (AA), and No answer (NA). Number of $U E, O E, A A$, and NA were calculated for all the domains in both the groups. Independent t-test was employed to find out the significant differences between the means of both the groups.

\section{Results}

The present study investigated the cognitive estimation abilities in healthy aged population. The results of descriptive statistics i,e total number of correct, Underestimation, Over-estimation and No answers for various domains in adults and geriatrics are depicted in table 1 and total number of correct, Under-estimation, Overestimation and No answers for independent domains in adults and geriatrics are depicted in table 2 .

Results of independent t-test showed that young adults were better in estimating the environment when compared to healthy aged population, which was significant at $\mathrm{t}(38)=2.057, \mathrm{p} \varangle .05$. Distance and size dimensions were remarkably affected in comparison to all other dimensions. When it comes to UE and OE, adult's scores were higher for UE than OE and vice versa for healthy aged individuals in all domains. Instances of No answers (NA) were higher in healthy aged population in comparison to young adults.

\begin{tabular}{|l|c|c|}
\hline Responses & Adults & Geriatrics \\
\hline Correct & 154 & 124 \\
\hline Under-Estimation & 114 & 84 \\
\hline Over-Estimation & 90 & 105 \\
\hline No Answer & 2 & 47 \\
\hline
\end{tabular}

Table 1 : Depicting the total number of correct, Under-estimation, Over-estimation and No answers for various domains in adults and geriatrics.

\begin{tabular}{|l|c|c|c|c|c|c|c|c|c|c|c|c|}
\hline Responses & \multicolumn{2}{|c|}{ Distance } & \multicolumn{2}{|c|}{ Quantity } & \multicolumn{3}{c|}{ Size } & \multicolumn{2}{c|}{ Weight } & \multicolumn{2}{|c|}{ Temperature } & \multicolumn{3}{|c|}{ Time } \\
\hline & $\mathrm{A}$ & $\mathrm{G}$ & $\mathrm{A}$ & $\mathrm{G}$ & $\mathrm{A}$ & $\mathrm{G}$ & $\mathrm{A}$ & $\mathrm{G}$ & $\mathrm{A}$ & $\mathrm{G}$ & $\mathrm{A}$ & $\mathrm{G}$ \\
\hline Correct & 12 & 5 & 30 & 23 & 33 & 23 & 19 & 22 & 17 & 11 & 40 & 36 \\
\hline Under-Estimation & 14 & 11 & 22 & 15 & 20 & 11 & 28 & 21 & 16 & 17 & 16 & 5 \\
\hline Over-Estimation & 33 & 26 & 8 & 14 & 7 & 18 & 13 & 5 & 7 & 5 & 23 & 30 \\
\hline No answer & 1 & 15 & 0 & 5 & 0 & 5 & 0 & 9 & 0 & 5 & 1 & 5 \\
\hline
\end{tabular}

Key: (A - Adults; G - Geriatrics)

Table 2: Depicting the total number of correct, Under-estimation, Over-estimation and No answers for independent domains in adults and Geriatrics.

\section{Discussion}

Traditionally, no attempt has been made to assess cognitive estimation in different domains (e.g., time vs. distance $)^{[8]}$.The present study attempted to include various dimensions in CET. The results of the present study indicated that healthy aged populations were poorer in estimating the environment in comparison to young adults. This could be due to the cognitive deterioration 
occurring as a part of healthy aging, which affects the performance of semantic abilities, memory etc. As cognitive estimation skill requires intact semantic abilities healthy aged population performed poorly on CET ${ }^{[9]}$. Individuals who perform poorly on the Cognitive estimation task also tend to have poorer semantic knowledge abilities and executive deficits, although these were not investigated in the present study. It is also reported that good relationship exists between cognitive estimation and tests of intelligence and memory ${ }^{[10]}$. Hence, it is natural to expect poorer performance on cognitive estimation in healthy aged population.

When it comes to individual dimensions, healthy aged population performed very poorly on the dimensions of distance and size in comparison to other dimensions such as time, length etc. This indicates that distance and size may be the first dimension to get affected on tests of cognitive estimation in comparison to other dimensions. These results could be due to the fact that these health aged individuals would undergo structural changes in the brain which might have caused poorer performance in cognitive estimation. It was found that the patient with frontal lobe lesion exhibited the difficulties in estimation abilities (size, weight, numerosity and time) ${ }^{[1]}$. This study showed that the mild damage to the frontal lobe resulted in gross estimation and verbal numerosity deficits with other cognitive abilities being near normal. Hence it can be assumed that these estimation abilities are more vulnerable for early regression. But, as suggested by few researchers, executive control processes shows robust differences in brain activity between older and younger subjects ${ }^{[3,13]}$. Therefore this difference in executive processes should have been reflected even in cognitive estimation abilities. Still there is no clarity on using these dimensions on CET as an early indicator for cognitive

\section{References}

1. Shallice T, Evans ME. The involvement of the frontal lobes in cognitive estimation. Cortex.1978; 14(2), 294-303.

2. Just MA, Carpenter PA. A capacity theory of comprehension: Individual differences in working memory. Psychological Review. 1992; 99, 122-149.

3. Jonides J, Marshuetz C, Smith EE., Reuter-Lorenz PA., Koeppe RA, Hartley A. Age differences in behavior and PET activation reveal differences in interference resolution in verbal working memory. deteriorations.

However, it may add to the arrays of instruments available for legal evaluation in Alzheimer's disease or any other pathological aging. It was reported that cognitive estimation to be a significant predictor of functional rehabilitation outcome in mild cognitive impairment ${ }^{[14]}$. Another author suggested that impaired estimation of numerical quantity in cognitive estimation test are not sufficient to situate at what level the deficit occurs (semantic representation of numbers, or translation from semantic representation of numbers to output, etc) ${ }^{[11]}$. Further studies are warranted in these aspects. Also, there is a need for further validation of the modified cognitive estimation test in the Indian population.

\section{Conclusion}

In the present study, cognitive estimation was investigated in young adults and healthy aged population. Results indicated that, the performance on CET was deviant in healthy aged population as compared to young adults, with pronounced difficulties in dimensions of distance and size. The results indicate that, the decline in cognitive estimation abilities is a part of healthy aging rather than a pathological condition. Thus CET can be used as an assessment tool in the assessment and management of cognitive disorders.

\section{Acknowledgement}

I take the privilege to convey my sincere gratitude to The HOD, Department of Audiology and Speech language Pathology, Kasturba Medical College, Mangalore, The Director, Nitte Institute of Speech and Hearing and The Principal, Nitte Institute of Speech and Hearing for their constant support and encouragement. I would like to extend my gratitude to the Participants for their support.

Journal of cognitive Neuroscience. 2000; 12(1), 188-196.

4. Levinoff EJ, Phillips NA, Verret L, Babins L, Kelner N, Akerib V, Chertkow H. Cognitive estimation impairment in Alzheimer disease and mild cognitive impairment. Neuropsychology.2006; 20(1), 123-132.

5. Shallice T, Evans ME. The involvement of the frontal lobes in cognitive estimation. Cortex. 1978; 14(2), 294-303.

6. Smith ML, Milner B. Differential effects of frontal-lobe lesions on cognitive estimation and spatial memory. Neuropsychologia. 1984; 
22, 697-705.

7. Jhonson MJ. That was your life: a biological approach to later life. An ageing population. M ilton Keynes: Open University Press. 1985.

8. Bullard ES, Fein D, Gleeson MK, Tischer N, Mapou RL, Kaplan E. The Biber Cognitive Estimation Test. Archives of Clinical Neuropsychology. 2004; 19, 835-846.

9. Brand M, Kalbe E, Kessler J. Qualitative and quantitative differences in cognitive estimation of patients with probable Alzheimer's diseasefrom healthy controls - what are the differences ? In P. Calabrese \& A.Neugebauer (Eds.), Memory and emotion . 2002; 425-429.

10. Freeman M R, Ryan JJ, Lopez SJ, M ittenberg W. Cognitive estimation in traumatic brain injury, relationships with measuresof intelligence, memory, and affect. International Journal of Neuroscience. 1995; 83, 269-273.

11. Revkin KS, Piazza M, Izard V, Zamarian L, Karner E, Delazer M. Verbal numerosity estimation deficit in the context of spared semantic representation of numbers: A neuropsychological study of a patient with frontal lesions. Neuropsychologia. 2008; 46, 2463-2475.

12. Nielson KA, Langenecker SA, Ross TJ, Garavan H, Rao SM, Stein EA. Comparability of functional MRI response in young and old during inhibition. Neuroreport. 2004; 15(1), 129-133.

13. Jackson JC, M itchella N, Hopkins RO. Cognitive Functioning, Mental Health, and Quality of Life in ICU Survivors: An Overview. Critical Care Clinics. 2009; 25(3), 615-628. 